\title{
A midline switch of receptor processing regulates commissural axon guidance in vertebrates
}

\author{
Homaira Nawabi, ${ }^{1,4}$ Anne Briançon-Marjollet, ${ }^{1,4}$ Christopher Clark, ${ }^{1}$ Isabelle Sanyas, ${ }^{1}$ \\ Hyota Takamatsu, ${ }^{2}$ Tatsusada Okuno, ${ }^{2}$ Atsushi Kumanogoh, ${ }^{2}$ Muriel Bozon, ${ }^{1}$ Kaori Takeshima, ${ }^{3}$ \\ Yutaka Yoshida, ${ }^{3}$ Frédéric Moret, ${ }^{1}$ Karima Abouzid, ${ }^{1}$ and Valérie Castellani ${ }^{1,5}$ \\ ${ }^{1}$ University of Lyon, University of Lyon 1, Claude Bernard Lyon1, CGMC, UMR, CNRS 5534, F-69000 Lyon, France; \\ ${ }^{2}$ Department of Immunology, Research Institute for Microbial Diseases, Osaka University, Osaka, 565-0871, Japan; ${ }^{3}$ Division \\ of Developmental Biology, Cincinnati Children's Hospital Medical Center, Cincinnati, Ohio 45229, USA
}

Commissural axon guidance requires complex modulations of growth cone sensitivity to midline-derived cues, but underlying mechanisms in vertebrates remain largely unknown. By using combinations of ex vivo and in vivo approaches, we uncovered a molecular pathway controlling the gain of response to a midline repellent, Semaphorin3B (Sema3B). First, we provide evidence that Semaphorin3B/Plexin-A1 signaling participates in the guidance of commissural projections at the vertebrate ventral midline. Second, we show that, at the precrossing stage, commissural neurons synthesize the Neuropilin-2 and Plexin-A1 Semaphorin3B receptor subunits, but Plexin-A1 expression is prevented by a calpain1-mediated processing, resulting in silencing commissural responsiveness. Third, we report that, during floor plate (FP) in-growth, calpain1 activity is suppressed by local signals, allowing Plexin-A1 accumulation in the growth cone and sensitization to Sema3B. Finally, we show that the FP cue NrCAM mediates the switch of Plexin-A1 processing underlying growth cone sensitization to Sema3B. This reveals pathway-dependent modulation of guidance receptor processing as a novel mechanism for regulating guidance decisions at intermediate targets.

[Keywords: Axon guidance; midline crossing; semaphorin; calpain; commissural neurons]

Supplemental material is available at http://www.genesdev.org.

Received June 5, 2009; revised version accepted December 18, 2009.

The developing neuronal projections navigate along highly diverse environments and manage complex pathway choices to reach their specific target tissues. Axon trajectories are specified by multiple cues, and guidance decisions crucially depend on regulatory mechanisms controlling in time and space the expression, distribution, and activity of the guidance machinery, including ligands, receptors, and signaling effectors ( $\mathrm{Yu}$ and Bargmann 2001). The pathfinding of long-distance projections proceeds in successive stages, with regularly positioned sources of attractants keeping axons in appropriate routes, referred to as intermediate targets. For example, in vertebrates, groups of neurons in the CNS send axon projections that navigate through a key intermediate target, the floor plate (FP), in which they cross the midline. In the developing spinal cord, commissural neurons reside in the dorsal horn, and their axons navigate

\footnotetext{
${ }^{4}$ These authors contributed equally to this work.

${ }^{5}$ Corresponding author.

E-MAIL castellani@cgmc.univ-lyon1.fr; FAX 33-0472442685.

Article is online at http://www.genesdev.org/cgi/doi/10.1101/gad.542510. Freely available online through the Genes \& Development Open Access option.
}

ventromedially to cross the midline and turn rostrally, extending along longitudinal pathways. Axon tracts then contact various spinal and higher-center neurons to establish circuits participating in the left-right control of sensory modalities and motor behaviors (Colamarino and Tessier-Lavigne 1995). Extensive studies established that Netrins, Semaphorins, IgSFCAMs, Slits, and various morphogens combine contact and diffusible attractive and repulsive effects to control commissural axon pathfinding (Augsburger et al. 1999; Garbe and Bashaw 2004; Dickson and Gilestro 2006).

A key issue that long-distance projections have to solve is how to leave an intermediate target. One possibility is that, among the guidance cues to which they are exposed, commissural axons first perceive attractants, thus being guided toward the intermediate target, and second repellents during in-growth, thereby receiving instructions for intermediate target exit. Accordingly, spinal commissural axons are attracted to the FP by Netrins and Shh, but then lose their responsiveness to Netrins and become sensitive to midline-derived repellents of the Slit, Semaphorin, and Ephrin families (Kidd et al. 1998; Brose et al. 1999; Zou et al. 2000; Imondi and Kaprielian 2001). Very 
little is known about the mechanisms through which these modulations are achieved. Suppression of Netrin attraction has been proposed to occur upon exposure to Slits, through the association of their respective DCC and Robo receptors (Stein and Tessier-Lavigne 2001). In flies, the sensitivity to Slits is silenced at the precrossing stage through a post-translational mechanism coupling the Slit receptor Robo to the Commissureless protein and targeting the complex for proteasomal degradation. This pathway is suppressed at the midline through a yet-unknown mechanism, thus enabling commissural axons to gain responsiveness to Slits (Keleman et al. 2002; Dickson and Gilestro 2006). However, the commissural-dependent mechanism has not proven effective in vertebrates, despite the conservation of Robo/Slit signaling at the ventral midline. Genetic approaches in mice indicated that a specific spliced variant of one of the three Robo genes, robo3, controls through a yet-unknown mechanism Robol/2 signaling and responsiveness to Slits (Chen et al. 2008). Another key but yet-unsolved issue is whether acquisition of responsiveness to guidance cues results from experienceindependent processes, with neurons being sensitized through an intrinsic mechanism, or rather from contextdependent pathways, with neurons being sensitized by signals localized at intermediate targets.

In this study, we explored the role of the midline repellent Semaphorin3B (Sema3B), a class 3 Semaphorin (Sema3) that was reported in in vitro assays to repel commissural axons after FP crossing (Zou et al. 2000). We show that crossing and post-crossing axon trajectories are defective in Sema3B-null embryos, consistent with a role for Sema3B in FP exit. We identify Plexin-A1 as the signaling coreceptor of Sema3B in this system, and show that Plexin-A1- and Sema3B-null embryos exhibit similar guidance defects of spinal commissural projections. We set up cultures of isolated FP tissue and dissociated commissural neurons and demonstrate that signals released by FP cells sensitize commissural growth cones to Sema3B. Naive commissural growth cones in culture and precrossing axon segments in vivo express the receptor subunit Nrp2, but Plexin-A1 level is very low. Upon exposure to FP signals conferring the response to Sema $3 \mathrm{~B}$, Plexin-A1 level is up-regulated and the protein distributes in the peripheral growth cone structures. In spinal cord explant cultures, Plexin-A1 expression was detected in axons emerging after FP crossing, while it was very low in explants cultures in which the FP was removed. When electroporated in the chick neural tube, the fluorescence of a Plexin-A1-gfpPhLuo fusion, allowing visualizing of a cell surface protein pool, is turned on upon FP crossing, which demonstrates that a switch of Plexin-Al level occurs in this intermediate target. Decreasing and increasing Plexin-A1 levels in cultured commissural neurons and in vivo is sufficient for altering growth cone responsiveness to Sema3B. Through various pharmacological and siRNA-based knockdown approaches, we show that the Plexin-A1 level is actively kept low at the precrossing stage due to the processing by calpain1, and that FP signals, by suppressing this protease pathway, enable accumulation of Plexin-A1 in commissural growth cones and sensitization to Sema3B. Consistently, calpain activity in unfixed embryonic spinal cord sections is detected in spinal neurons and precrossing axon segments, but not in crossing axon segments and FP cells. Pharmacological inhibition of calpain activity in vivo induces defects of FP in-growth and premature turning. Biochemical approaches demonstrated that this calpain activity directly processes Plexin-A1 at the precrossing stage. Last, we identify an active FP component, the Ig superfamily cell adhesion molecule NrCAM, in the switch of PlexinA1 processing, triggering commissural axon sensitization to Sema3B.

\section{Results}

Sema3B/Plexin-A1 signaling is required for commissural axon guidance at the midline

We first examined Sema3B and Nrp2 expression in embryonic day 11.5 (E11.5) and E12.5 developing mouse spinal cords by in situ hybridization. Consistent with previous work (Zou et al. 2000), Sema3B was found expressed at the ventral midline and in dorsal territories, while Nrp2 mRNA was detected in the dorsal horn where commissural neurons reside (Fig. 1A; Supplemental Fig. S1A). These expression patterns were consistent with a role of Sema3B in the guidance of commissural projections. Next, we analyzed the trajectory of commissural axons in Sema3B-null mutant mice. Crossing and post-crossing commissural pathways were examined in spinal cord open book preparations by insertion of DiI (1,1'-dilinoleyl-3,3,3' $3^{\prime}$ '-tetramethylindocarbocyanine, 4-chlorobenzenesulfonate) crystals in the domain of commissural cell bodies (Fig. 1B). In contrast to axons that turned rostrally after midline crossing in the wild-type embryos in $80 \%$ of cases, axons from Sema3B-null embryos exhibited aberrant trajectories in $60 \%$ of cases, ranging from stalling or turning in the FP, caudal instead of rostral turning, and dorsally rather than ventrally directed growth after FP crossing (number of crystals/number of embryos: $72 / 19$ for $+/+, 47 / 12$ for $+/-$, and $90 / 13$ for $-/-$, from four litters) (Fig. 1C). In another set of experiments, we focused on FP-crossing phenotypes and compared the proportion of DiI-labeled tracts that could exit the FP (number of crystals/number of embryos: 52/ six for $+/+, 28 /$ three for $+/-$, and $51 /$ four for $-/-$, from two litters) (Fig. 1D). More than $60 \%$ exited the FP in the wild-type embryos for only $32 \%$ in the null embryos. Thus, the genetic ablation of Sema3B disrupts the behavior of crossing and post-crossing commissural axons. FP and interneuron markers were not modified by the loss of Sema3B, suggesting that these defects reflected a requirement for Sema3B guidance activity at the ventral midline (Supplemental Fig. S1B,C). The Sema3 signaling is mediated by Plexin-A family members that are obligatory Nrp coreceptors for the activation of transduction cascades (Kruger et al. 2005; Bechara et al. 2008). Until now, there was no information regarding the identity of the Plexin-A recruited to Nrp2 for mediating Sema3B effects in axon guidance. Based on expression patterns in in situ hybridization performed on E12.5 coronal sections, Plexin-A1 

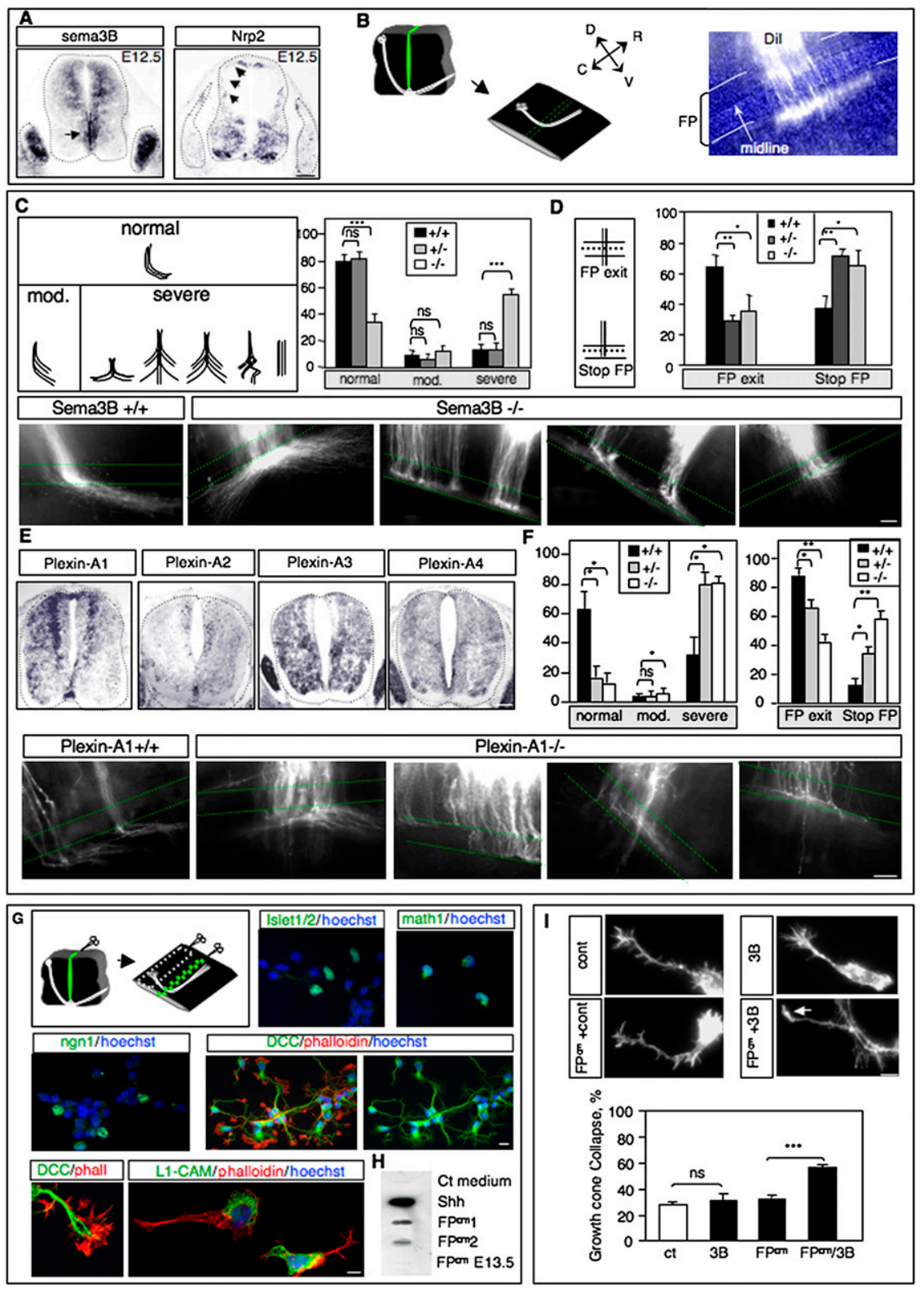

Figure 1. Crossing and post-crossing commissural defects in Sema3B and Plexin-A1 knockout embryos, and gain of response to Sema3B induced by $\mathrm{FP}^{\mathrm{cm}}$. (A) In situ hybridization on E12.5 cross-sections showing Sema3B transcripts at the ventral midline and Nrp2 in dorsal spinal cord domain of commissural neurons (arrows). (B) Schematic drawing of the spinal commissural pathway and DiI labeling in open book preparations. Green dashed lines delineate the FP positioned by phasecontrast observations. $(C)$ Illustrations of commissural tracts in wild-type embryos $\left(S_{e m a} B^{+/+}\right)$and various misrouting in the Sema3B-null embryos $\left(S_{e m a 3 B^{-1-}}\right)$, stalling or turning in the $\mathrm{FP}$, aberrant caudal turning, and dorsally directed growth. $(C)$ Classification of commissural trajectories in normal, mild, and severe cases for

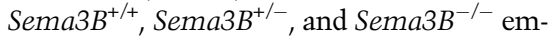
bryos. $(D)$ Classification focused on axon behaviors within the FP showing that, at E12.5, significantly less axon tracts exited the FP in the $+/-$ and $-/-$ embryos than in the $+/+$ embryos. $(E)$ In situ hybridization on E12.5 cross-sections to label Plexin-A1, Plexin-A2, Plexin-A3, and Plexin-A4 transcripts in the spinal cord. Plexin-A1 mRNA is strongly detected in spinal interneurons. $(F)$ Illustrations and diagrams of DiI labeling of commissural projections in open book preparations from Plexin- $A 1^{+/+}$and Plexin- $A 1^{-/-}$embryos, showing aberrant crossing and post-crossing trajectories in the Plexin-A1-null embryos. Bars, $100 \mu \mathrm{m}$. (G) Schematic drawing of the tissue from which dissociated neuronal cultures and isolated FP tissue culture were performed. Cultured neurons express ngn 1 , math 1 , and islet $1 / 2$ transcription factors, and cell surface DCC markers of commissural neurons. The L1-CAM marker is restricted to the soma in axon and growth cone compartment. $(H)$ Slot blots showing immunodetection of the FP cue Shh in two $\mathrm{FP}^{\mathrm{cm}}$ samples at E12.5 and its down-regulation at E13.5. (I) Histogram and microphotographs showing that $\mathrm{FP}^{\mathrm{cm}}$ triggers a Sema3Binduced collapse response. Bar: $A, B, 15 \mu \mathrm{m}$.

was the strongest candidate, as its transcript was detected in dorsal interneurons, while that of the other Plexin-As were not or were only weakly and/or uniformly distributed in the spinal cord (Fig. 1E; Cheng et al. 2001). We then examined spinal commissural projections in PlexinA1-null embryos. DiIs were placed in open book preparations at stage E12.5/E13 (number of crystals/number of embryos: 54/six for +/+, 61/eight for $+/-$, and 84/seven for $-/-$, from five litters) (Fig. 1F). Errors of commissural axon trajectories were detected and, remarkably, they mimicked those identified in the Sema3B-null embryos, with premature turning, stalling in the FP, defasciculation, and caudal instead of rostral turning before and after crossing (Fig. 1F). FP and dorsal markers also were not affected by Plexin- $A 1$ genetic ablation (Supplemental Fig. S2A,B). This analysis supported that Plexin-A1 is required for Semaphorin3B-mediated axon guidance in the FP.

\section{Local FP signals confer responsiveness to Sema3B}

Explants assays established that commissural axons acquire responsiveness to Sema3B upon FP crossing (Zou et al. 2000). We confirmed this result in cocultures of open book explants with cell aggregates secreting Sema3B, observing that the growth of commissural axons toward cells secreting Sema3B was permitted when the FP was removed, but was inhibited in intact preparations 
(five embryos, $>15$ explants/condition, three independent experiments) (Supplemental Fig. S3A). We hypothesized that the gain of response results from exposure to local signals present in the FP. To address this hypothesis, we set up cultures of isolated FP tissue to produce conditioned medium $\left(\mathrm{FP}^{\mathrm{cm}}\right)$, and assessed it in a model of dissociated dorsal spinal neurons. To validate this model, we examined the expression of different commissural neuron markers (Fig. 1G). First, neurons composing the cultures expressed Islet1/2, math1, and ngn1 transcription factors characteristic of the $\mathrm{dl} 1, \mathrm{dl} 2$, and $\mathrm{dl} 3$ pools of commissural neurons. Second, the cell surface commissural marker DCC was detected in the axons and growth cones of all cultured neurons (Fig. 1G). In vivo, L1-CAM is expressed by commissural neurons, but is present only along crossing and post-crossing axon segments (Supplemental Fig. S3B). In our model, L1-CAM was detected in the soma and, strikingly, it was excluded in most cases from the axon and the growth cone compartments, and only occasionally $\mathrm{L}^{+}$growth cones were detected (Fig. 1G; Supplemental Fig. S3B). Lastl initial axon outgrowth was dependent on netrin-1, as expected for commissural neurons (data not shown). Thus, cultured dissociated dorsal neurons express the markers of commissural neurons at the precrossing stage. To verify that the tissue put in culture was composed by FP cells, we assessed the presence of known soluble FP-derived cue Shh and Netrin-1 in slot blots, and could detect their presence in $\mathrm{FP}^{\mathrm{cm}}$ samples (Fig. 1H; Supplemental Fig. S3C).

Next, the neuronal cultures were exposed to either control, Sema3B, or $\mathrm{FP}^{\mathrm{cm}}$ treatment, or in combined application (Fig. 1I). Notably, the level of collapse was increased significantly by combined application of Sema3B and $\mathrm{FP}^{\mathrm{cm}}$, but not $\mathrm{FP}^{\mathrm{cm}}$ or Sema3B alone (number of cones per condition/number of experiments: 560/seven for control, 560/seven for Sema3B, 1040/13 for FP $\mathrm{cm}^{\mathrm{cm}}$, and 1280/16 for $\mathrm{FP}^{\mathrm{cm}}$-Sema3B) (Fig. 1I). A similar gain of collapse was observed when the $\mathrm{FP}^{\mathrm{cm}}$ was removed prior to Sema3B application, indicating that the $\mathrm{FP}^{\mathrm{cm}}$ and Sema3B does not act in synergy, but rather that the $\mathrm{FP}^{\mathrm{cm}}$ contains signals that capacitate commissural neurons to be responsive to Sema3B (Supplemental Fig. S3D). Commissural neurons isolated at E11.5, E12.5, and E13.5 all failed to collapse upon Sema3B exposure, indicating that neurons are unlikely to acquire responsiveness through maturation, at least in these culture conditions (Supplemental Fig. S3E).

\section{Plexin-A1 level is up-regulated by FP signals} in commissural growth cones

We hypothesized that responsiveness to Sema3B could be gained through regulation at the receptor complex level. Changes in expression levels, availability, or spatial distribution of some subunits could allow the growth cone to assemble a functional receptor only within the FP. To test this idea, we first examined the distribution of the receptor subunits in spinal commissural axons by immunohistochemistry on E11.5 cross-sections. The specificity of the anti-Plexin-Al antibodies used for the study was controlled in transfected cells and in cross-sections of
Plexin-A1-null embryos (Supplemental Fig. S4A,B). Nrp2 could be detected along both precrossing, crossing, and post-crossing axon shafts (Fig. 2A). Interestingly, PlexinA1 expression was very weak in precrossing segments, but was strongly up-regulated in crossing and post-crossing segments (Fig. 2A). This raised first the possibility that commissural neurons are unresponsive to Sema3B due to the lack of Plexin-Al in the growth cone, and second that Plexin-Al expression and/or distribution could be modified during FP in-growth to allow commissural axons acquiring responsiveness to Sema3B. We thus investigated whether the $\mathrm{FP}^{\mathrm{cm}}$ in our culture assay could regulate the expression of Plexin-A1. Interestingly, in the basal condition, Plexin-Al was present in the central domain, but absent from the peripheral growth cone structures, playing key roles in the perception of extracellular signals. Remarkably, $\mathrm{FP}^{\mathrm{cm}}$ application induced Plexin-Al spreading in the growth cone filopodia (Fig. 2B). These observations were confirmed by counting of Plexin-A1 clusters (Fig. 2B). We found that the number of Plexin-A1 clusters increased by only $16 \%$ in the central domain, but by $160 \%$ in the filopodia. This was not due to morphological modifications, since the number of filopodia per growth cone was statistically comparable in all conditions (Fig. 2B). Thus, FP signals induce Plexin-A1 to accumulate in the filopodia.

We also asked whether the Plexin-A1 level is regulated by the $\mathrm{FP}^{\mathrm{cm}}$ by performing quantitative fluorescence measurement, and found significant increase of the total Plexin-A1 pool in the soma and the growth cone (Fig. $2 \mathrm{C}, \mathrm{D})$. This result was confirmed with a second antiPlexin-A1 antibody recognizing a different Plexin-A1 epitope (Supplemental Fig. S4A). A similar increase was also found when the quantitative analysis was restricted to the surface pool of Plexin-A1 (Fig. 2D). Such an increase was not observed for another Plexin-A family member, Plexin-A2 (Fig. 2E). Nrp2 was also not obviously modified after exposure to FP signals (Supplemental Fig. S5). We assessed the Plexin-A1 level with a complementary biochemical approach (Fig. 2F). Fresh dorsal spinal cord tissue was isolated for ex vivo stimulation by $\mathrm{FP}^{\mathrm{cm}}$ and control treatments, and was processed for Western blot analysis. As expected, the Plexin-A1 level was increased by twofold in the $\mathrm{FP}^{\mathrm{cm}}$ condition compared with the control, while the Nrp2 level remained comparable (Fig. 2F). To provide direct evidence that Plexin-A1 upregulation takes place in axons that cross the FP, spinal cord explants in which the FP was either removed or left intact were cultured in a three-dimensional substrate and labeled with anti-DCC and anti-Plexin-A1 antibodies (Fig. 2G). Plexin-A1 and DCC levels were measured in growth cones that had or had not crossed the FP. We found that the DCC level remained constant in both conditions, while the Plexin-A1 level increased by $36 \%$ in growth cones that crossed the $\mathrm{FP}$, thus demonstrating that axons must cross the FP to up-regulate Plexin-A1 (-FP, 64 growth cones; +FP, 34 growth cones; two independent experiments).

Next, we used the chick embryo to investigate the regulation of the Plexin-Al level upon FP crossing in vivo. To validate this animal model for our question, we 


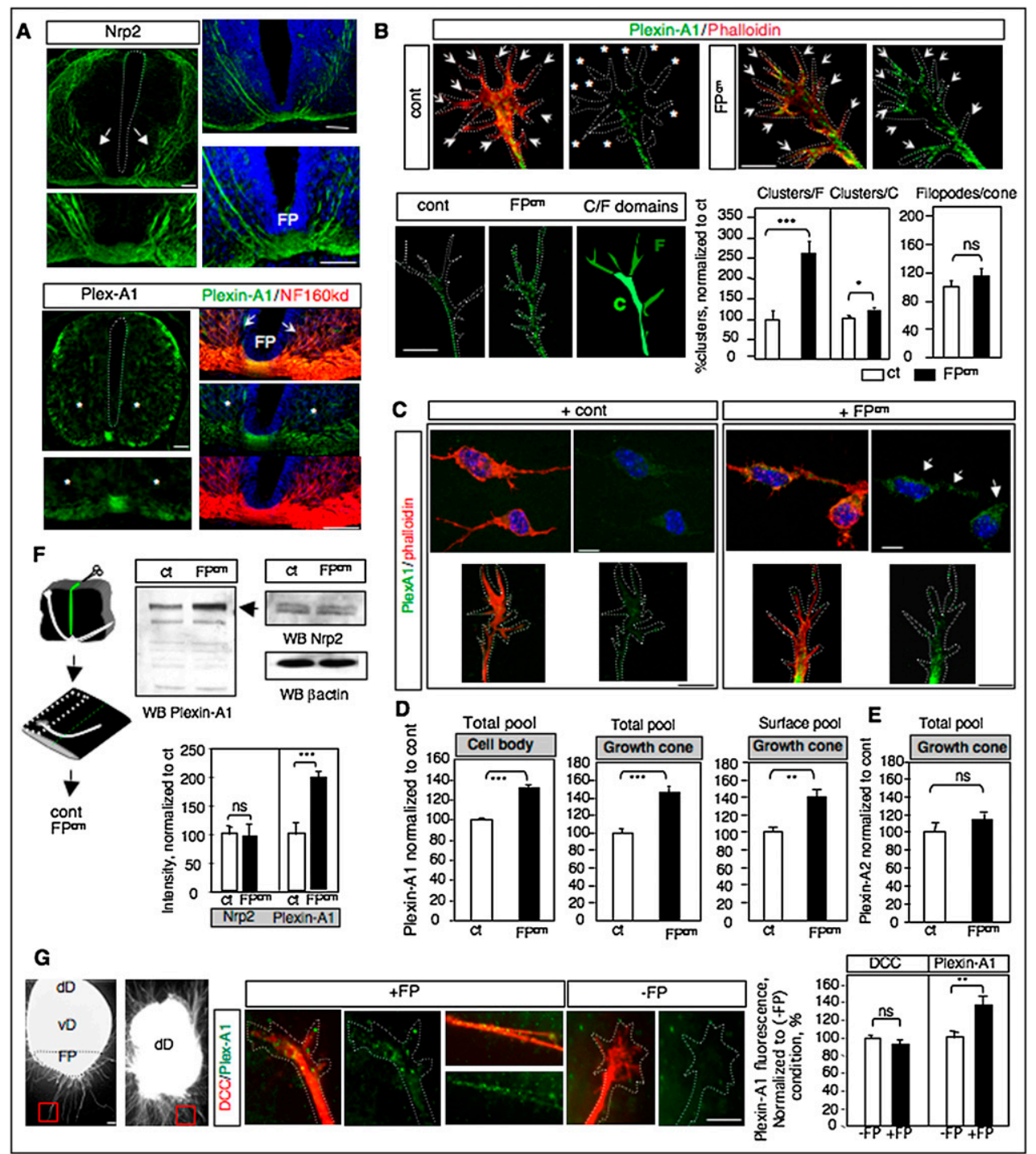

Figure 2. The Plexin-A1 level is increased by FP signals. (A) Immunolabeling of the Sema3B receptor subunits Nrp2 and Plexin-A1 (green) in E11.5 cross-sections with nuclear counterstaining (blue). Nrp2 is expressed on precrossing segments. Plexin-Al is initially very low and is up-regulated in crossing and post-crossing segments. Bars, $100 \mu \mathrm{m}$. $(B)$ Microphotographs illustrating the difference of Plexin-Al filopodial distribution between control and $\mathrm{FP}^{\mathrm{cm}}$ conditions (white arrows and stars). Counting of Plexin-A1 clusters in the central and peripheral growth cone domains. Treatment with $\mathrm{FP}^{\mathrm{cm}}$ induces a strong increase of PlexinAl clusters in the peripheral domain without modifying the number of filopodia per growth cone. Increase of Plexin-Al clusters in the central domain is very modest. Bar, $15 \mu \mathrm{m} .(C)$ Illustrations of Plexin-Al immunolabeling (in green, phalloidin in red) in neuronal cultures showing Plexin-A1 increase in the $\mathrm{FP}^{\mathrm{cm}}$ condition, in the soma, and in the growth cone. Bar, $15 \mu \mathrm{m}$. $(D)$ The histograms summarize the quantitative analysis of Plexin-A1 fluorescence in the soma and the growth cones (total pool and cell surface) and its increase by FP signals. (E) Plexin-A2 fluorescence in the growth cones is not up-regulated by FP signals. $(F)$ Western blot analysis of the Plexin-A1 level in fresh dorsal spinal tissue stimulated with control (cont) and $\mathrm{FP}^{\mathrm{cm}}$. (Left panel) Experimental procedure. (Right panel) Rise of the Plexin-A1 but not the Nrp2 level in Western blot. The histogram shows quantification of band intensity and the increase of Plexin-A1 but not Nrp2 in the $\mathrm{FP}^{\mathrm{cm}}$ condition, compared with control. $(G)$ Explants assays with spinal cord tissue in which the FP was removed or was not removed. Illustration and quantification of DCC and Plexin-A1 growth cone fluorescence in commissural axons that crossed or did not cross the FP. Bar, $15 \mu \mathrm{m}$. (dD) Dorsal domain of the spinal cord; (vD) ventral domain of the spinal cord.

confirmed that chick commissural neurons are unresponsive to Sema3B at the basal level, but acquire responsiveness to Sema3B upon exposure to chick $\mathrm{FP}^{\mathrm{cm}}$ (Supplemental Fig. S6). To visualize the cell surface expression of Plexin-A1, the coding sequence was fused to gfp pHLuorin, a Ph-sensitive gfp that fluoresces in compartments with neutral $\mathrm{pH}$ such as the cell membrane, but very weakly in acidic intracellular compartments (Jacob et al. 2005). After transfection of the vector encoding Plexin-A1-Phluo in COS7 cells, the fluorescence was almost restricted to the cell surface, contrasting with a control egfp fusion membrane protein that was, in addition, detected in intracellular 
compartments; Fig. 3A). Next, Plexin-A1PhLuo was electroporated at very low levels $(0.5 \mu \mathrm{g} / \mu \mathrm{L})$ in the chick neural tube. Under these conditions, Plexin-A1-PhLuo was detected in dividing neuroepithelial cells and post-mitotic cells settling in the mantle (Fig. 3B). In commissural neurons, the fluorescence was visible in the soma and the proximal axon segment but was very weak in the precrossing axon segment, thus indicating that the PlexinA1-PhLuo level was successfully repressed at the precrossing stage. Remarkably, intense fluorescence was detected in growth cones and axon segments after FP crossing (total of six embryos, $100 \%$ penetrance of the observation) (Fig. 3C). This compartmentalized pattern was never observed in embryos electroporated with egfp (total of six embryos) (Fig. 3D). To quantify these observations, Plexin-A1-PhLuo and egfp were coelectroporated with $r f p$ (Fig. 3E). In the Plexin-A1-PhLuo condition, commissural growth cones were red prior to FP crossing, becoming yellow in the FP, while in the egfp condition, growth cones were already yellow before FP entry (Fig. 3E).

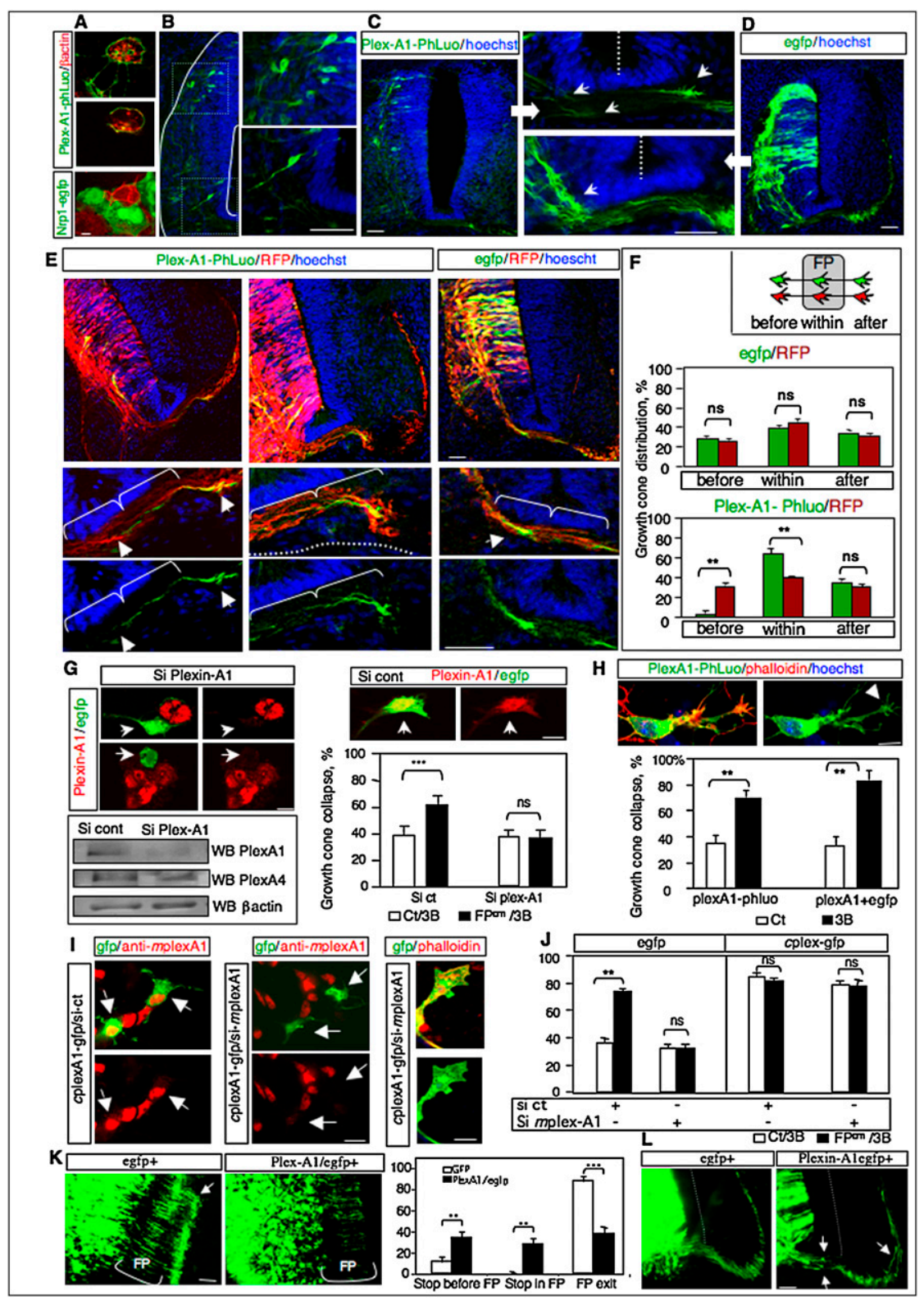

Figure 3. Plexin-Al up-regulation in commissural growth cones during FP crossing and gain of response to Sema3B. (A) Two Z scans showing restricted Plexin-A1-PhLuo cell surface expression in COS7 cells. $(B-D)$ Electroporation of Plexin-A1PhLuo or egfp in the chick neural tube. Plexin-A1-PhLuo is visible in polarized neuroepithelial cells, lateral post-mitotic soma, and initial axon segments, but is weakly detected along precrossing commissural axons, compared with egfp (white arrows). Note the bright fluorescence of Plexin-A1-PhLuo after FP crossing (right white arrow). (E) Plexin-A1$P h L u o / R F P$ and egfp/RFP coelectroporation. (Left panel) Yellow growth cones are detected before crossing in the egfp/RFP but not the Plex-A1-PhLuo/RFP condition. In the right panels, Plexin-A1-PhLuo green fluorescence is switched on upon FP crossing, unlike rfp, which is detected before FP entry. $(F)$ Distribution of green and red growth cones before FP in-growth, in the FP, and after FP exit in the Plex-A1-PhLuo/RFP and egfp/RFP conditions, showing the extremely low number of green Plex-A1-PhLuo growth cones at the precrossing stage. Bar, $100 \mu \mathrm{m}$. (G) Microphotographs of siRNAmediated knockdown of Plexin-A1 expression (red) in cultured dorsal spinal neurons labeled with gfp (green). Bar, $15 \mu \mathrm{m}$. Western blot showing the knockdown of PlexinA1 but not Plexin-A4 by the si-Plex-A1, and the lack of effect of the si-control (si-cont). The histogram shows the loss of $\mathrm{FP}^{\mathrm{cm}}$. induced collapse responsiveness to Sema3B in the Plexin-Al knockdown condition. (H) Plexin-A1-PhLuo overexpressed in commissural neurons is present at the soma and growth cone surface. Histogram showing that overexpressed Plexin-A1-PhLuo and vsv-Plexin-A1 both confer to commissural growth cones responsiveness to Sema3B. Bar, $15 \mu \mathrm{m}$. (I) Rescue of Plexin-A1 restores the sensitivity to Sema3B. Illustrations of combined siRNA and gfp-Plexin-A1 cotransfection showing that mouse Plexin-A1 (mPlex-A1) was knocked down (labeled with mouse-specific anti-Plexin-A1) and replaced by chick gfp-Plexin-A1 (cPlex-A1, gfp label). $c$ Plex-A1 is present in the growth cones. Bar, $20 \mu \mathrm{m}$. (J) The histograms show the results of a collapse assay and the rescue by $c$ Plex-A1. $(K)$ Illustrations and quantifications of DiI labeling in open book preparations after electroporation of Plexin-A1/egfp or egfp at high concentration in the chick embryo. Plexin-Al but not egfp overexpression induced defects of FP crossing. (White arrow in the egfp condition) Post-crossing tract. Bar, $100 \mu \mathrm{m}$. $(L)$ Gfp staining in cross-sections illustrating growth cone stalling at the FP entry in the Plexin-Albut not egfp condition. (White arrows) Stalling growth cones and aberrant post-crossing axon trajectories. Bar, $100 \mu \mathrm{m}$. 
We counted the number of growth cones in the red and green channels before FP entry, within the FP, and after FP exit in the Plex-A1-PhLuo/rfp and egfp/rfp conditions (total of 73 sections from six embryos, 587 growth cones). The number of growth cones distributed equally before and after FP crossing in the egfp/rfp controls. In striking contrast, the number of green but not red fluorescent growth cones in the Plex-A1-Phluo condition was strongly shifted to the crossing and post-crossing categories (Fig. $3 F)$. Thus, in vivo, the Plexin-Al cell surface level is kept very low in commissural growth cones at the precrossing stage and is up-regulated during FP in-growth.

If the Plexin-Al level is central to the regulation of growth cone responsiveness, then modifying Plexin-A1 levels should alter commissural growth cone behaviors. Accordingly, knockdown should invalidate the sensitization, and overexpression should mimic the effect of FP signals and confer responsiveness to Sema3B. First, PlexinA1 was knocked down in cultured neurons by siRNA transfection. Efficiency and specificity were controlled by immunolabeling in cultured commissural neurons and in Western blot (Fig. 3G). Analysis of growth cone behaviors showed that those extending from neurons in which Plexin-A1 was silenced were unable to acquire responsiveness to Sema3B upon exposure to $\mathrm{FP}^{\mathrm{cm}}$ (50 neurons analyzed per condition, two independent experiments) (Fig. 3G). Second, the Plexin-A1 level was increased by overexpression of Plexin-A1-PhLuo in cultured commissural neurons (Fig. 3H). Intense fluorescence was detected in the soma and growth cone compartment, demonstrating the ectopic accumulation of overexpressed Plexin-A1 at the growth cone surface. In this condition, the growth cones were found responsive to the collapsing effect of Sema3B in the absence of $\mathrm{FP}^{\mathrm{cm}}$. As control for Plexin-A1PhLuo functionality, similar results were obtained after transfection of a vsv-tagged Plexin-A1 form (Supplemental Fig.S7A). Similar results were obtained in neurons isolated from the chick embryo (Supplemental Fig.S7B). We also performed rescue experiments (Fig. 3I). siRNA directed against endogenous mouse Plexin-A1 (mPlexA1) was cotransfected with chick Plexin-A1-egfp fusion (cPlexA1), which escapes the silencing. Knockdown and overexpression was confirmed by immunolabeling with a Plexin-A1 antibody recognizing $m$ PlexAl but not $c$ PlexAl, and gfp observations to detect $c$ PlexA1. We observed in a collapse assay that $c$ PlexA 1 could rescue the sensitivity of neurons in which endogenous mPlexA1 was abolished (Fig. 3J). As observed in the overexpression-alone condition, the sensitization was no longer dependent on $\mathrm{FP}^{\mathrm{cm}}$. Thus, an artificial increase of the Plexin-Al level mimics the effect of the $\mathrm{FP}^{\mathrm{cm}}$ and is sufficient for conferring responsiveness to Sema3B. We also examined the consequences of knockdown of another Plexin-A, Plexin-A3, which appeared to be expressed by commissural neurons. Nevertheless, siRNA transfection, which efficiently and specifically extincted Plexin-A3, did not prevent commissural axon responses to Sema3B (Supplemental Fig. S7C).

We next assessed the consequences of in vivo PlexinA1 overexpression. Plexin-A1 and egfp constructs were overexpressed by electroporation in the chick neural tube (total of 24 embryos, 240 crystals). Given that Sema3B is produced at the midline, but also along the precrossing axon route, premature responsiveness should affect the growth of precrossing axons and their entry in the FP. The pattern of commissural projections was first examined by insertion of DiI in the dorsal domain of open book preparations (Fig. 3K). In embryos overexpressing Plexin-A1/egfp but not egfp alone, a significant proportion of commissural axons failed to reach and enter the FP (Fig. 3K; Supplemental Fig. S7D). Transversal sections were also performed to visualize the trajectory of individual egfp ${ }^{+}$ axons. Consistent with the in toto DiI analysis, growth cones accumulated at the FP entry in the Plex-Al/egfp ${ }^{+}$ but not egfp ${ }^{+}$condition (Fig. 3L). This phenotype was thus consistent with the acquisition of the precrossing commissural response to the FP-repellent Sema3B.

\section{FP signals suppress proteolytic calpain1 activity to increase Plexin-A1 level}

Next, we explored the metabolic pathways by which FP signals trigger increase of the Plexin-Al level. We assessed activation of protein synthesis and, conversely, inhibition of proteasomal degradation, as both of these pathways were described to regulate guidance receptor levels at the midline (Brittis et al. 2002; Garbe and Bashaw 2004). We took a pharmacological approach in our culture assay, and quantified the Plexin-Al level in the soma and the growth cone compartments. Application of MG132, a proteasome inhibitor, had no detectable effect on the Plexin-A1 level in both the soma and the growth cone (Fig. 4A). Surprisingly, inhibition of protein synthesis with anisomycin prevented the $\mathrm{FP}^{\mathrm{cm}}$-triggered gain of Plexin-Al in the soma, but notably not in the growth cone (Fig. 4B,E). Thus, these pathways are unlikely to control the increase of Plexin-A1 levels by FP signals. We then assessed the possible implication of proteolytic processing by first applying a cocktail of inhibitors that blocks a panel of proteases, such as serine proteases, cysteine proteases, and metalloproteases (Fig. $4 \mathrm{C})$. Interestingly, in the soma and the growth cone, this treatment increased Plexin-Al and induced its accumulation in the filopodia in the control condition, fully mimicking the $\mathrm{FP}^{\mathrm{cm}}$ condition. Moreover, combined pharmacological and $\mathrm{FP}^{\mathrm{cm}}$ treatments did not produce additive effects, thus likely affecting a common pathway. We investigated the nature of the protease and found that specific inhibition of the cysteine protease calpain 1 with ALLn $(1 \mu \mathrm{M})$ recapitulated the effect of the cocktail (Fig. $4 \mathrm{D}, \mathrm{E})$. In contrast, Nrp2 level was not found increased by calpain1 inhibition (Supplemental Fig. S8A). The presence of calpain1 in commissural neurons was confirmed by immunolabeling (Fig. 4F). If calpain1 maintains PlexinA1 at low levels, then its inhibition should confer responsiveness to Sema3B. Calpain1 was thus knocked down with siRNA transfected in neuronal cultures. Efficiency and specificity of the siRNAs were controlled in immunolabeling and Western blots (50 neurons per condition, two independent experiments) (Fig. 4G; Supplemental Fig.S8B). Analysis in collapse assays demonstrated 


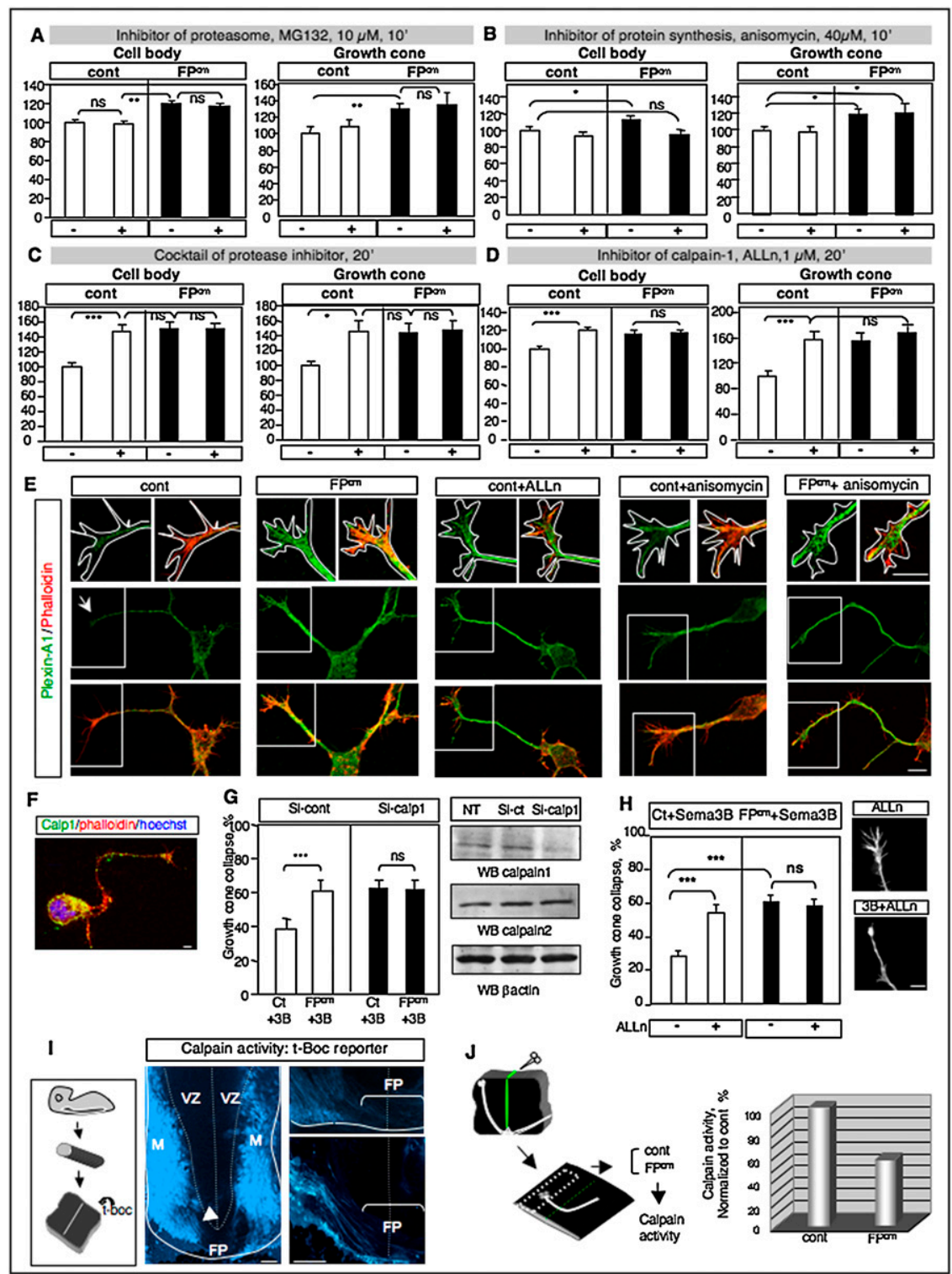

Figure 4. The Plexin-Al level is regulated by a calpain1-dependent protease pathway. (A) Application of the proteasome inhibitor MG132 has no effect on Plexin-A1 levels, both in control and $\mathrm{FP}^{\mathrm{cm}}$ conditions. $(B)$ Inhibition of protein synthesis with anysomycin blocked $\mathrm{FP}^{\mathrm{cm}}$-induced Plexin-A1 up-regulation in the soma but had no effect in the growth cone. Note that applications were shortened to $10 \mathrm{~min}$ to limit cytotoxicity. $\mathrm{FP}^{\mathrm{cm}}$-induced increase of Plexin-Al is attenuated but still significant. $(C)$ A cocktail of protease inhibitors increased the Plexin-A1 level in the soma and the growth cone when applied in the basal condition, but not with $\mathrm{FP}^{\mathrm{cm}} .(D)$ Inhibition of calpain1 had similar effects. $(E)$ Illustrations of neuronal cultures showing that protein synthesis inhibition does not prevent $\mathrm{FP}^{\mathrm{cm}}$. induced increase of Plexin-Al (green) in the growth cone. Illustrations of increase of Plexin-A1 and accumulation in the filopodia (green) induced by the calpainl inhibitor ALLn as with the $\mathrm{FP}^{\mathrm{cm}}$, compared with control. Anisomicyn treatment does not prevent Plexin-A1 accumulation in the $\mathrm{FP}^{\mathrm{cm}}$ condition. Soma and growth cones are delineated with phalloidin-TRITC. Bar, $15 \mu \mathrm{m}$. (F) Coimmunolabeling of calpain1 (green) and phallodin (red) in cultured commissural neurons. Bar, $15 \mu \mathrm{m}$. (G) Calpain1 knockdown experiments with siRNA. Immunoblots of neuroblastoma cell lysates showing the efficiency of the si-calp but not the si-control (si-cont) to silence endogenous calpain1. Calpain-2 level is not altered by the si-calp. The histogram shows acquisition of growth cone collapse response to Sema3B in the basal condition induced by calpain 1 silencing. $(H)$ Collapse assay showing that calpain1 inhibition with ALLn $(1 \mu \mathrm{M})$ conferred responsiveness to Sema3B in the basal condition (control). Bar, $15 \mu \mathrm{m}$. (I) Endogenous calpain activity in unfixed chick spinal cord sections using t-Boc. The fluorescence is detected in the mantle (M) but not in the ventricular zone (VZ) and the FP. The magnification illustrates the sharp decrease of fluorescence in commissural axons entering the FP. Bar, $100 \mu \mathrm{m}$. (J) Measure of endogenous calpain activity in dorsal spinal tissue stimulated with $\mathrm{FP}^{\mathrm{cm}}$. The histogram presents the decreased rate of calpain 1 activity in tissue treated with $\mathrm{FP}^{\mathrm{cm}}$ normalized to control condition.

that application of the calpain1 inhibitor ALLn $(1 \mu \mathrm{M})$ in the neuronal cultures conferred responsiveness to Sema3B, in the absence of $\mathrm{FP}^{\mathrm{cm}}$ (number of growth cones per condition: 155 for control, 126 for control/ALLn, 123 for FP, and 127 for FP/ALLn; two independent experiments) (Fig. 4H). Knockdown of calpain1 with siRNA resulted in similar effects (Fig. $4 \mathrm{H}$ ).

These data suggested that calpains might be active in commissural neurons to process Plexin-A1 at the precrossing stage but not in crossing axons, due to the action of the FP signals. To address this issue, we first investigated the pattern of calpain activity in the developing chick spinal cord (Fig. 4I). Spinal cords were isolated from
HH25 (Hamburger and Hamilton 25) embryos, rapidly sectioned into $200-\mu \mathrm{m}$-thick slices; incubated for $15 \mathrm{~min}$ with the calpain activity reporter t-Boc, whose cleavage produces blue fluorescence (Robles et al. 2003); and observed immediately with a confocal microscope. As expected, intense fluorescence was detected in the mantle where post-mitotic spinal neurons reside. In contrast, any fluorescent staining was detected in the ventricular zone containing the progenitors and the FP. Notably, although the t-Boc fluorescence in cell populations along the commissural pathway prevented assessment of calpain activity in commissural axons in the dorsal and intermediate spinal cord domains, at more ventral positions, 
t-Boc fluorescence was very clear in precrossing fibers, abruptly disappearing in crossing axon segments at all confocal $Z$ positions (Fig. 4I).

Second, we measured endogenous calpain activity in fresh dorsal spinal cord tissue after exposure to control and $\mathrm{FP}^{\mathrm{cm}}$ treatments using a biochemical approach that, unlike t-boc staining, allowed quantitative analysis. Consistent with our model, decrease of calpain activity by $52 \%$ was found in the samples stimulated with $\mathrm{FP}^{\mathrm{cm}}$ compared with the controls, and the decrease could be reproduced in four independent experiments (Fig. 4J).

If calpain activity is required for precrossing commissural axon guidance, its inhibition should produce guidance defects at the FP. To address this issue, intraperitoneal injections of MDL28170 (or calpain inhibitor III), a calpain inhibitor that crosses the blood-brain barrier and efficiently inhibits calpain activity in the CNS (Markgraf et al. 1998; Mingorance-Le Meur and O'Connor 20091, were performed in pregnant mice at successive E10.5 and E11.5. Commissural axon trajectories were

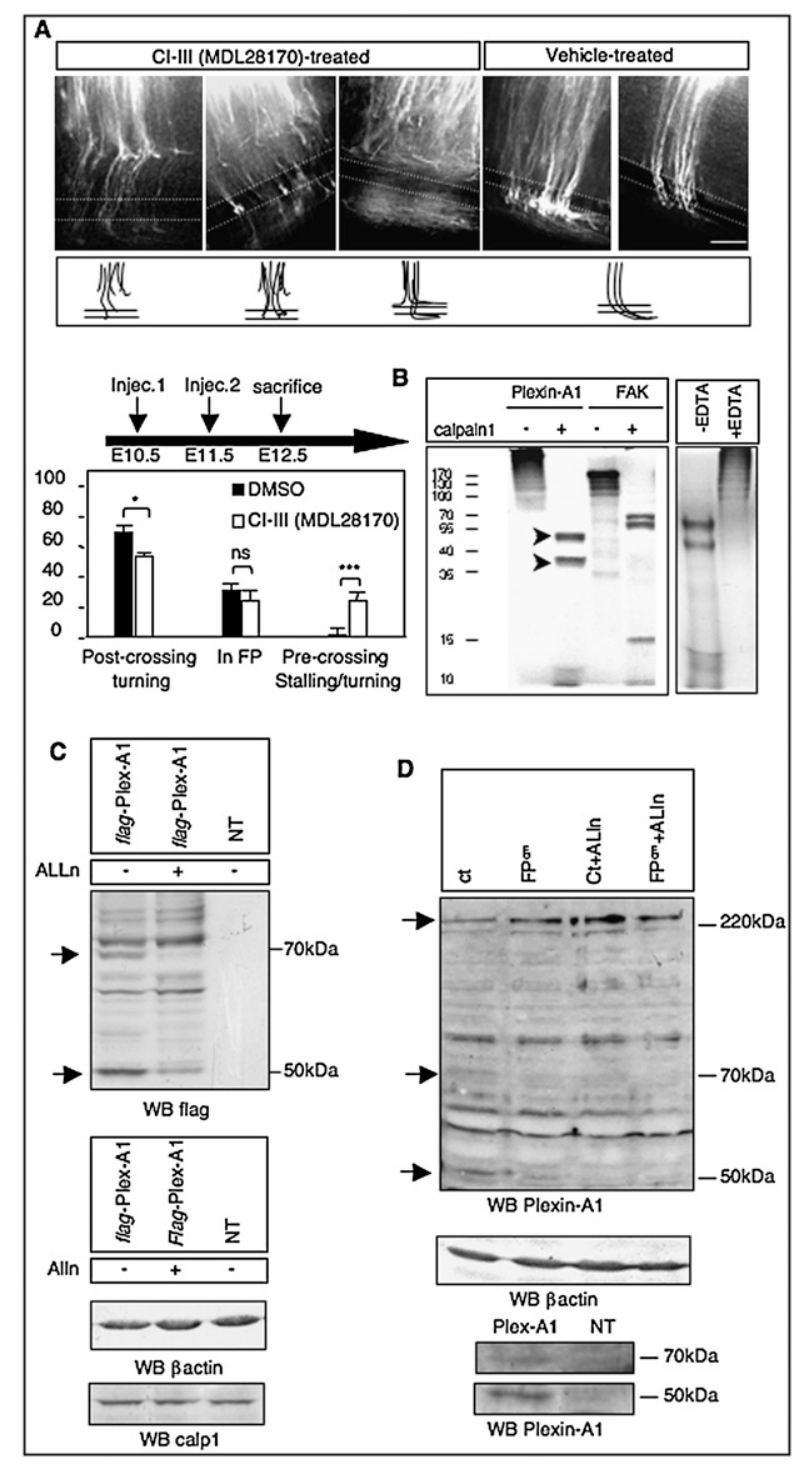

analyzed in open book preparations of E12.5 embryos (number of crystals/number of embryos: 73/six for vehicle and 204/nine for MDL28170) (Fig. 5A). In the vehicletreated animals, commissural axons reached the FP in almost all cases, whereas in the MDL28170-treated animals, commissural axons stopped growing, turned in both directions, or stalled at the FP entry in $25 \%$ of the cases. The crossing and post-crossing trajectories of axons that could reach the FP were not deeply modified, and axons turned in an appropriate direction after FP crossing. Thus, calpain activity is required for commissural axons to reach and enter the FP, consistent with our finding that calpain activity silences precrossing commissural responsiveness to the FP cue Sema3B.

Finally, we examined whether Plexin-A1 is processed through direct or indirect action of calpain1. First, we performed an in vitro cleavage assay (Fig. 5B). Plexin-A1 was translated in vitro in the presence of MetS35, and the product was incubated with purified active calpain 1 and analyzed in Western blot. We found that Plexin-A1 is cleaved into two major fragments of apparent molecular masses of $55 \mathrm{kDa}$ and $45 \mathrm{kDa}$. Inhibition of calpain 1 activity by EDTA blocked the processing. Positive control of calpain activity was done with a known calpain target, the focal adhesion kinase (FAK). Second, Plexin-A1 tagged in Nter with Flag was transfected in COS7 cells. The cells were incubated by ALLn or control treatment, and Plexin-A1 band patterns were compared in Western blot (Fig. 5C). We observed two Plexin-A1 bands at $\sim 70$ $\mathrm{kDa}$ and $50 \mathrm{kDa}$ in the control condition that were strongly reduced when calpain 1 activity was inhibited with ALLn. Similar band patterns were observed using N-ter vsv-Plexin-A1 fusion and gfp-Plexin-A1 fusion (data not shown). Differences of masses between these two assays likely reflected post-translational modifications in cell lines. Thus, the Plexin-A1 extracellular domain is processed by calpains. Third, we examined whether such processed fragments could be detected in spinal dorsal

Figure 5. Calpain is required for precrossing commissural axon guidance and cleaves Plexin-1. (A) Illustrations and quantifications of DiI labeling in open book preparations. Inhibition of calpain activity induces significant defects of precrossing axon behaviors, with axons failing to enter the FP or turning before crossing. Bar, $100 \mu \mathrm{m}$. (B) Autoradiography of Western blot showing that in vitro translated Plexin-Al is cleaved by active calpain1 into two main fragments. Positive control: FAK. PlexinAl is not processed when calpain1 is inhibited by EDTA. $(C)$ Western blot showing that Flag-Plexin-A1 transfected in COS7 cells is processed by endogenous calpain1 into two fragments. The fragments are reduced by ALLn application. (Right panel) Western blot to detect calpain1 and ßactin in the same samples. (D) Western blot showing Plexin-A1 processing in fresh dorsal tissue in control and $\mathrm{FP}^{\mathrm{cm}}$ conditions. Inhibition of calpain 1 by ALLn in the control condition induced accumulation of fulllength Plexin-A1 and reduction of the processed fragments. The band patterns of control + ALLn and $\mathrm{FP}^{\mathrm{cm}}$ are similar. ALLn application in the $\mathrm{FP}^{\mathrm{cm}}$ condition does not modify the band pattern. (Bottom panel) Plexin-A1-processed fragments are detected with the same anti-PlexinAl antibody in transfected COS cells but not in nontransfected control COS7 cells (NT). 
tissue (Fig. 5D). Dorsal spinal cords were dissected from E12.5 embryos, and were treated with control and $\mathrm{FP}^{\mathrm{cm}}$ in the presence and absence of ALLn. Plexin-A1 band patterns were compared in Western blot using an antibody recognizing Plexin-A1 extracellular epitopes. Notably, in the control condition, the full-length Plexin-A1 band was strongly increased by ALln, while conversely, the processed fragments that we found present in the samples were strongly decreased. As expected, the PlexinA1 band pattern of $\mathrm{FP}^{\mathrm{cm}}$ and control + ALLn was similar, and the presence of ALln in the $\mathrm{FP}^{\mathrm{cm}}$ did not modify the band pattern (Fig. 5C). Thus, these experiments provided evidence that calpain 1 activity in spinal commissural neurons cleaves Plexin-A1, and that this processing is suppressed by FP signals.

\section{The FP cue NrCAM regulates Plexin-A1 level and commissural responsiveness to $S e m a 3 B$}

In the next step, we explored the nature of the active components mediating the gain of response to Sema3B. NrCAM was an interesting candidate due to its high and restricted expression in the FP, and its functional properties to regulate axon growth and guidance during the formation of various commissural tracts (Fig. 6A; Falk et al. 2005; Williams et al. 2006). Moreover, metalloprotease-mediated release of

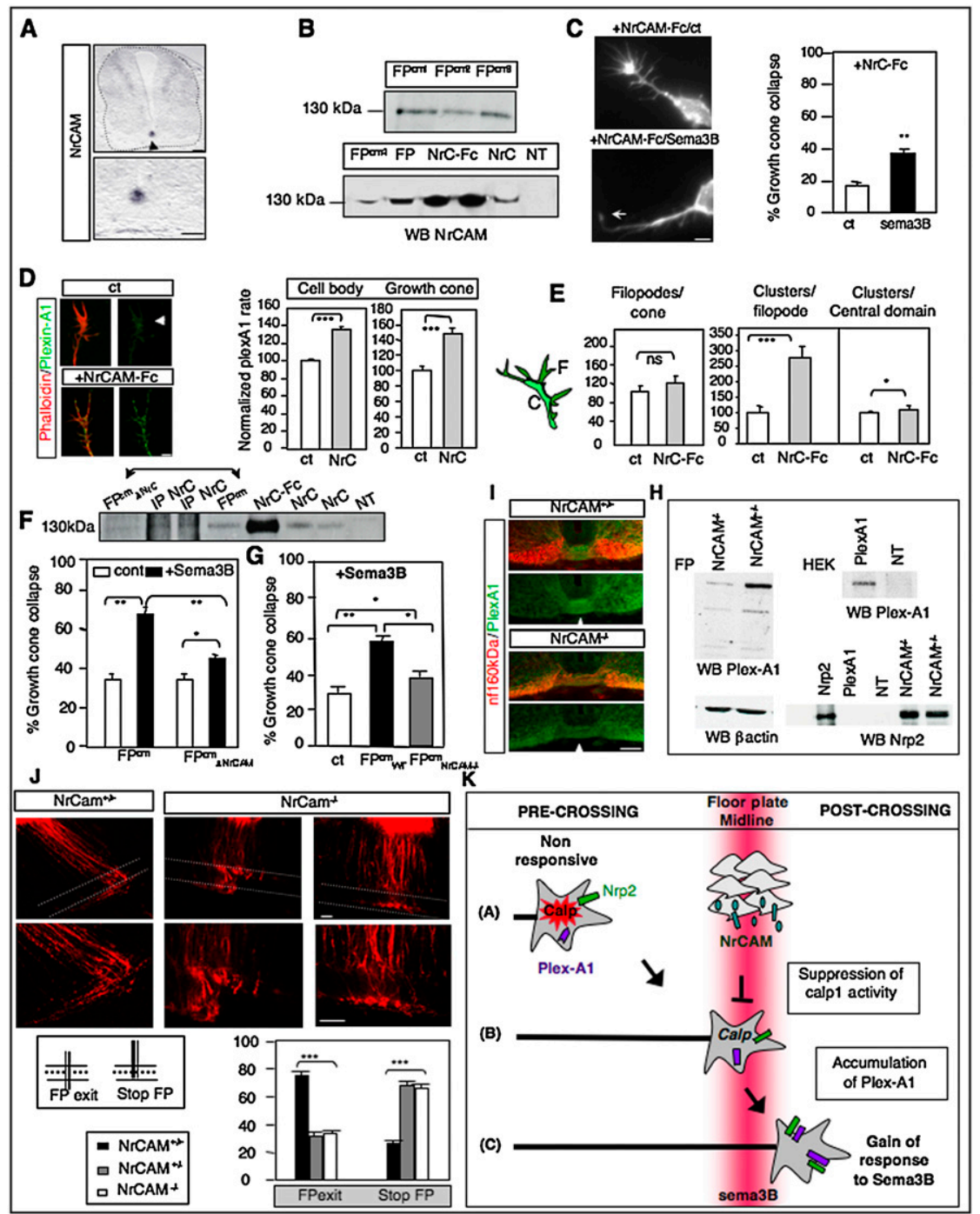

Figure 6. NrCAM is an active $\mathrm{FP}$ cue in the regulation of the Plexin-Al level and acquisition of responsiveness to Sema3B. (A) In situ hybridization on E12.5 cross-sections showing mRNA transcript of NrCAM in the FP. (B) Western blot showing detection of NrCAM in four independent samples of $\mathrm{FP}^{\mathrm{cm}}$ and in $\mathrm{FP}$ extracts $(\mathrm{FP}) .(C)$ Collapse assay with chimeric NrCAM-Fc. Application of NrCAM-Fc combined with Sema3B allows commissural neurons to acquire responsiveness to Sema3B, while NrCAM-Fc, when applied alone, has no effect. $(D)$ Application of NrCAM-Fc triggers a significant increase of Plexin-A1 levels in the soma and growth cones of cultured commissural neurons. $(E)$ Plexin-A1 clusters accumulate in the growth cone peripheral domain upon exposure to NrCAM-Fc, while the number of filopodia per growth cone remains comparable. $(F)$ NrCAM was biochemically depleted from the $\mathrm{FP}^{\mathrm{cm}}$ through immunoprecipitation with anti-NrCAM antibody (compare bands pointed to by the arrow). The Western blot shows strong reduction of $\mathrm{NrCAM}$ detection in this sample $\left(\mathrm{FP}^{\mathrm{cm}}{ }_{\triangle \mathrm{NrCAM}}\right)$ compared with native $\mathrm{FP}^{\mathrm{cm}}$. In functional collapse assays, the efficiency of $\mathrm{FP}^{\mathrm{cm}}{ }_{\triangle \mathrm{NrCAM}}$ to trigger responsiveness to Sema3B was significantly decreased, compared with native $\mathrm{FP}^{\mathrm{cm}}$. In the experiments using $\mathrm{FP}^{\mathrm{cm}}{ }_{\Delta \mathrm{NrCAM}}$, the control (cont) medium was also preincubated with an antibody to normalize possible nonrelevant effects of the biochemical depletion. (G) FP was isolated from the NrCAM mutant mouse line to produce $\mathrm{FP}^{\mathrm{cm}}$. This medium was significantly less efficient than the wildtype $\mathrm{FP}^{\mathrm{cm}}$. (H) Immunohistochemical labeling of E12.5 cross-sections showing a decrease of Plexin-Al reactivity in the FP of $\mathrm{NrCAM}^{-/-}$embryos, compared with wild-type embryos. (I) The Plexin-A1 level in FP extracts from $\mathrm{NrCAM}^{+/+}$and $\mathrm{NrCAM}^{-/-}$embryos was compared in Western blot. NrCAM deficiency induced a strong decrease of the Plexin-A1 level, while the Nrp2 level remained constant. (J) Analysis of commissural projections in open book preparations from the NrCAM mouse line. The microphotographs and histogram show that NrCAM deficiency induced significant defects of FP crossing, with axons stalling in the FP. Bar: $A, H, J, 100 \mu \mathrm{m} ; C, D, 15 \mu \mathrm{m}$. (K) Model for the mechanisms controlling the acquisition of Sema3B responsiveness at the ventral midline. (Panel $A$ ) At the precrossing stage, active calpainl cleaves the signaling coreceptor Plexin-Al and silences Sema3B responsiveness. (Panel $B$ ) During FP in-growth, commissural axons are exposed to FP signals that suppress calpainl activity. (Panel C) Plexin-A1 is allowed to accumulate in the growth cones, thus sensitizing them to Sema3B. This guidance response participates in the FP exit. 
active NrCAM ectodomain has been reported, making it possible that soluble NrCAM contributes to the property of the $\mathrm{FP}^{\mathrm{cm}}$. We thus assessed the presence of NrCAM in the $\mathrm{FP}^{\mathrm{cm}}$ by Western blot and could detect, with an antibody recognizing NrCAM extracellular epitopes, a $130-\mathrm{kDa} \mathrm{NrCAM}$ form corresponding to NrCAM ectodomain (Fig. 6B). We next assessed the functional properties of NrCAM ectodomain in our neuronal assay. Soluble NrCAM-Fc was applied to commissural neurons. While the level of collapse was very low in the presence of NrCAM-Fc alone, it significantly increased when NrCAM was applied with Sema3B, indicating that NrCAM-Fc could trigger responsiveness to Sema3B (Fig. 6C). As expected, NrCAM-FC application could also increase Plexin-A1 level in commissural cell bodies and growth cones, and the protein accumulated in the growth cone peripheral domain (Fig. 6D,E). Next, to determine whether NrCAM contributes to the property of the $\mathrm{FP}^{\mathrm{cm}}$, NrCAM was depleted from the medium by immunoprecipitation with an anti-NrCAM antibody. As controlled by Western blot, this procedure strongly reduced the NrCAM level in the $\mathrm{FP}^{\mathrm{cm}}$ (Fig. 6F). This $\mathrm{FP}^{\mathrm{cm}}{ }_{\Delta \mathrm{NrCAM}}$ was applied to commissural neurons, and its efficiency in triggering growth cone responsiveness to Sema3B was significantly attenuated (Fig. 6F). We also assessed the consequence of $N r C A M$ genetic ablation by producing $\mathrm{FP}^{\mathrm{cm}}$ from $\mathrm{NrCAM}$-null embryos. Consistently, the effect of the $\mathrm{FP}^{\mathrm{cm}}$ was almost totally abrogated, indicating that NrCAM is a major active component of the $\mathrm{FP}^{\mathrm{cm}}$ (Fig. 6G). To validate these data, we investigated whether NrCAM deficiency alters Plexin-A1 level in the FP. First, immunolabeling was performed on E12.5 $\mathrm{NrCAM}^{+/+}$and $\mathrm{NrCAM} \mathrm{M}^{-/-}$embryonic cross-sections. We observed a decrease of Plexin-A1 labeling in the FP of the NrCAM-null embryos compared with wild-type embryos (Fig. 6H). Second, FP tissue was dissected from $\mathrm{NrCAM}^{-/-}$and $\mathrm{NrCAM}^{+/+}$embryos originating from the same littermates, and the Plexin-A1 band pattern was analyzed in Western blot. The PlexinA1 band was strongly decreased in the samples from $N r C A M^{-1-}$ embryos compared with those of the wildtype embryos, while, as control, Nrp2 level remained similar (Fig. 6I). By altering the Plexin-A1 level and subsequently the gain of response to Sema3B, NrCAM deficiency would then be expected to impair FP exit. Consistently, in open book preparations labeled with DiI, we found that a significant proportion of axon tracts stalled in the FP of the $N r C A M^{-/-}$embryos instead of exiting the FP to turn rostrally, as observed in $\mathrm{NrCAM}^{+/+}$ embryos (Fig. 6J).

\section{Discussion}

The present study identifies a pathway-dependent mechanism in vertebrates by which spinal commissural axons acquire sensitivity to a midline-derived repellent of the Semaphorin family. Sema3s share typical receptor organization in multimolecular complexes (Kruger et al. 2005). We found that precrossing commissural axons are maintained unresponsive to Sema3B by the protease calpain1, processing the signaling moiety of the Sema3B receptor complex, Plexin-A1, to prevent expression of the integral protein in commissural growth cones. Exposure to the local FP signal NrCAM suppresses this processing, enabling full-length Plexin-A1 accumulation in crossing commissural growth cones, thus switching on their responsiveness to Sema3B (Fig. 6K).

\section{Semaphorin3B/Plexin-A1 signaling regulates guidance decisions at the ventral midline}

Several ligand/receptor pairs regulate commissural axon guidance at the midline, playing synergic and complementary roles (Garbe and Bashaw 2004). First, Netrin/DCC and Shh/BOC attract commissural axons toward the FP (Okada et al. 2006). Repulsive Slit/Robo signaling ensures that axons exit the FP and do not cross the midline again. Nevertheless, genetic loss of Robo signaling (Robo1 and Robo2) does not totally abolish FP exit, thus indicating that other cues participate in this process (Dickson and Gilestro 2006). Recent work identified SCF1 as an additional cue whose growth-promoting effect helps commissural axon exiting the FP (Gore et al. 2008). Ephrin-Bs are other candidates, particularly Ephrin-B3, which is expressed by the FP (Kadison et al. 2006). Zou et al. (2000) showed that important guidance decisions in the FP are mediated by the Semaphorin receptor Nrp2. We found that, in Sema3B-null mutant mice, commissural axons often stall in the FP or grow at aberrant directions after midline crossing. These defects are very similar to those observed in the Nrp2 knockouts, thus showing that Sema3B might be the Nrp2 ligand required in vivo for proper commissural guidance at the midline. Nevertheless, despite their common expression at the ventral midline, Slits and Sema3B chemorepellents likely have specific roles, since the recrossing of commissural axons characterizing the loss of Robo/Slit signaling was not detected in the Sema3B knockouts. We could identify Plexin-A1 as the Plexin-A family member mediating Sema3B responses in spinal commissural axons. Likewise, Plexin-A1 is synthesized by commissural neurons, and its invalidation by siRNA abolishes the Sema3B-induced collapse response of their growth cones, normally triggered by FP signals. Moreover, Sema3B- and Plexin-A1null embryos develop similar guidance defects of commissural projections at the FP.

\section{Processing of guidance receptor by calpains: a mechanism for silencing Sema3B responsiveness at precrossing stage}

In Drosophila, responsiveness of precrossing commissural axons to Slit is silenced through coupling of the Slit receptor Robo to Commissureless, and sorting for proteasome degradation (Dickson and Gilestro 2006). In vertebrates, a spliced variant of one of the three Robo gene products, Robo-3.1, appears as a functional equivalent of Drosophila Commissureless, preventing through a yetundetermined mechanism Robol and Robo2 from mediating responsiveness to Slits at the precrossing stage (Sabatier et al. 2004; Chen et al. 2008). The present study 
highlights a novel mechanism by which precrossing commissural responses are silenced, based on processing of guidance receptors.

An interesting aspect of this pathway is that it does not prevent ligand/receptor interaction as for the Robo/Slit pair in Drosophila and possibly in vertebrates, since Nrp2 sorting to the growth cone surface is not prevented. Rather, it precludes accumulation of full-length signaling moiety of the receptor complex specifically transducing Sema3B in these neurons. This mechanism is advantageous and well-suited to the Semaphorin signaling, as it enables the other receptor subunit, Nrp2, to engage in other complexes.

Calpains are calcium-dependent cysteine proteases, regulating various processes. For example, they play pivotal roles in cell motility and synaptic functions by cleaving components of adhesion complexes and neurotransmitter receptors (Carragher and Frame 2002; Wu and Lynch 2006). Their functions during neuronal development are less characterized, but calpains are present in neuronal growth cones, are activated by intracellular calcium transients to reduce growth cone motility, and also are found downstream from Semaphorin5B (To et al. 2007). Interestingly, calpains process rather than degrade proteins, and are seen as regulators of protein functions, modulating protein-protein interactions, phosphorylation state, distribution, and traffic (Carragher and Frame 2002). Our data indicate that calpain 1 is active in commissural neurons and maintains the integral form of Plexin-A1 at very low levels at the precrossing stage by cleaving the Plexin-A1 extracellular domain, generating two fragments that can be detected in Western blot. This processing could take place before protein sorting to the cell membrane. Likewise, previous studies reported detection of calpain in the lumen of endoplasmic reticulum and golgi vesicles, thus being at an appropriate location for cleaving neosynthesized target proteins during their intracellular traffic (Hood et al. 2004, 2006). Alternatively, Plexin-A1 could be cleaved at the cell surface, since increasing evidence indicates that calpains are externalized and can be retained to the membrane surface through association with proteoglycans (Abe et al. 1998; Nishihara et al. 2001; Frangie et al. 2006; Peltier et al. 2006). Several of our results support an important role for calpain activity during precrossing commissural axon guidance. First, in the spinal cord sections, calpains were found active in spinal neurons and precrossing commissural axon segments. Second, inhibition of calpain activity in vivo resulted in strong defects of commissural axon behaviors before FP crossing: axons failing to enter the FP, turning before FP crossing, or even not reaching the FP. Interestingly, all of these defects were also induced by Plexin-A1 overexpression in the chick embryo, thus supporting that calpain-1 activity prevents Plexin-A1 expression at the precrossing stage. Whether this protease suppresses precrossing commissural responses to other midline repellents by processing guidance receptors other than Plexin-A1, such as Robo1/2 or Eph receptors, is an intriguing possibility that will be assessed in future studies.
Commissural axons acquire Sema3B responsiveness through a pathway-dependent mechanism, implicating local FP signals

Our results showed that suppression of calpain activity in the FP is instrumental for the gain of commissural responsiveness to Sema3B. Several data support this conclusion. First, in unfixed spinal cord sections, calpain were not active in FP cells or in the crossing axon tract. Second, we found that the FP tissue released signals that inhibit calpain activity in spinal tissue. Third, suppression of calpain activity could increase integral Plexin-A1 levels in acute dorsal tissues and cultured commissural neurons and induce responsiveness to Sema3B.

Commissural axons were found to acquire responsiveness to a variety of repellents, but whether this occurs through a pathway-dependent or pathway-independent mechanism remains unclear. In the Xenopus visual system, the temporal switch from attractive to repulsive behavior of retinal axons to Netrin1 does not depend on pathway experience (Shewan et al. 2002). Our data do not support the view that such an experience-independent mechanism operates to confer responsiveness to Sema3B at the midline. First, dorsal spinal neurons isolated from early E11 to late E13.5 were equally unresponsive to exogenous Sema3B application (Supplemental Fig. S3B). Second, in open book preparations, removing the FP was sufficient to abolish the repulsive behavior of commissural neurons to a focal source of Sema3B. Finally, neurons became sensitive to Sema3B upon exposure to $\mathrm{FP}^{\mathrm{cm}}$, independent from the developmental stage at which they were collected. Our data thus support that signals emanating from intermediate target cells play pivotal roles in the switch of responsiveness.

Consistently, we could show that the Ig superfamily cell adhesion molecule NrCAM is an active FP component, regulating the Plexin-A1 level and acquisition of responsiveness to Sema3B. High levels of $N r C A M$ transcripts were detected in the FP, and the protein was present in the $\mathrm{FP}^{\mathrm{cm}}$. In our neuronal assay, soluble NrCAM mimicked the $\mathrm{FP}^{\mathrm{cm}}$, triggering responsiveness to Sema3B and increase of the PlexinA1 level. In contrast, neither Netrin-1 nor Shh could recapitulate the gain of responsiveness to Sema3B conferred by the $\mathrm{FP}^{\mathrm{cm}}$ (H Nawabi and $\mathrm{V}$ Castellani, unpubl.). Moreover, the biochemical or genetic depletion of NrCAM strongly altered the properties of the $\mathrm{FP}^{\mathrm{cm}}$. In vivo, strong decrease of the Plexin-A1 level in the FP of NrCAM-null embryos was found and significant amounts of axon tracts abnormally stalled in the FP. NrCAM is expressed by various developing neuronal projections, and several previous studies implicated it in the regulation of axon navigation, as receptors or coreceptors for environmental guidance cues (Lustig et al. 2001; Falk et al. 2005; Williams et al. 2006). Likewise, NrCAM interaction with Nrp2 was found to be required for axons to normally form the anterior commissure in the brain, and NrCAM expression is required by RGC axons to form proper patterns of ipsilateral/contralateral commissures in the visual system (Falk et al. 2005; Williams et al. 2006). Interestingly, NrCAM is highly expressed in specialized 
glial structures in the ventral midline-not only in the spinal cord, but also in all upper floors of the CNS (Lustig et al. 2001)-but how NrCAM glial sources contribute to axon pathfinding remains unknown. The present work establishes a novel function for $\mathrm{NrCAM}$ as an intermediate target cue regulating the expression level of guidance receptors in the growth cones to control pathway choices at the ventral midline.

\section{Materials and methods}

\section{Genotyping and vector cloning}

Genotyping was performed as described in Falk et al. (2005), Yoshida et al. (2006), Takegahara et al. (2006), and Sakurai et al. (2001). Mouse Plexin-A1-PhLuorin was generated by introducing in Nter the coding sequence of the PhLuorin cloned from a vector encoding GABA A-PhLuorin-gfp (Jacob et al. 2005). Gfp was fused in Nter of the coding sequence of chick Plexin-A1.

\section{Western blot}

Spinal cords from E12.5 embryos were prepared in an open book configuration. Tissues were treated with control supernatant and $\mathrm{FP}^{\mathrm{cm}}$ with or without ALLn $(10 \mu \mathrm{M}$; Sigma $)$ for $30 \mathrm{~min}$. Dissected tissues were lyzed in $2 \times$ laemmli buffer. Cos7 cells transfected (Exgen, Euromedex) with Flag-plexinA1, vsv-Plexin-A1, and gfpPlexin-A1 were treated for $1 \mathrm{~h}$ with ALLn (10 $\mu \mathrm{M}$; Sigma). Samples were analyzed by Western blot using goat anti-Nrp2 (1/1000; R\&D), rabbit anti-plexA1 (1/1000; AbCAM), anti-Calpain1 and anti-Calpain2 (1/500, Santa Cruz Biotechnologies), antiNrCAM (1/1000; AbCAM), anti-vsv (1/1000; Sigma), anti-Flag $(1 / 1000)$, and anti- $\beta$ actin (1/1000; Sigma) antibodies.

Cocultures, explant cultures, cultures of dissociated neurons, and collapse assay

FPs were isolated from E12.5 embryos and cultured in threedimensional plasma clots (Castellani et al. 2000) in B27-supplemented Neurobasal medium (Gibco). The supernatant was collected after 48 h. For coculture experiments, HEK 293 cells were transfected with plasmids encoding either Sema3B-Alcaline Phosphatase fusion protein or control Alcaline Phosphatase. Cell aggregates were cocultured with spinal cord open books as described in Falk et al. (2005). Cocultures and spinal cord explants with or without FP were grown for 24 or 48 h, fixed in $4 \%$ paraformaldehyde (PFA), and stained with phalloidin-TRITC or with an anti-neurofilament antibody, or with anti-DCC antibody (BD Bioscience) and anti-PlexinAl antibody (Santa Cruz Biotechnologies). Axon outgrowth was quantified by measuring axon length using Image J software. Plexin A1 and DCC fluorescence levels were quantified with Image J software, as in Moret et al. (2007). For the collapse assay, dorsal spinal cord tissues from E11.5, E12.5, or E13.5 embryos were dissociated, and cells were plated into polylysin- and laminin-coated glass coverslips in Neurobasal supplemented with B27, glutamine (Gibco), and Netrin-1 (R\&D) medium. After 1 or $2 \mathrm{~d}$ in vivo (DIV), neurons were incubated with control or $\mathrm{FP}^{\mathrm{cm}}$ or different molecules for $30 \mathrm{~min}$ at $37^{\circ} \mathrm{C}$. Then Sema3B-AP was added on cells for $30 \mathrm{~min}$ at $37^{\circ} \mathrm{C}$. Cells were fixed in PFA $4 \% / 1.5 \%$ sucrose and labeled with phalloidin-TRITC (1/500; Sigma). Collapsed growth cones were scored as in Falk et al. (2005). The following were used: protease inhibitor cocktail (Sigma), calpain inhibitor I,

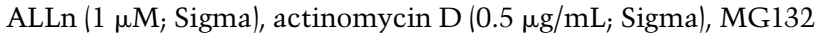

(10 $\mu \mathrm{M}$; Sigma), anisomycin (40 $\mu \mathrm{M}$; Sigma), and Shh $(2 \mu \mathrm{g} / \mathrm{mL}$; Sigma). siRNA to Plexin-A1, calpain1, and control siRNA (Santa Cruz Biotechnologies) were transfected with Lipofectamin2000 according to the manufacturer's recommendations (Invitrogen). Statistical comparisons were done with Student's $t$-test for collapse assays and ANOVA for analysis of fluorescence level: $\left(^{\star}\right) P$-value $\left.<0.05 ;\left.\right|^{\star \star}\right) P$-value $<0.01 ;\left(^{\star \star \star}\right) P$-value $<0.001$.

\section{Immunohistochemistry, in situ hybridization, and $t$-Boc assay}

Immunolabeling of neuronal cultures and sections was performed with antibodies anti-Plexin-A1 (1/100; Santa Cruz Biotechnologies, AbCAM, Chemicon), anti-Plexin-A2 (1/100; Santa Cruz Biotechnologies), anti-Nrp2 (1/100; R\&D), anti-L1-CAM (1/100; Chemicon), anti-Ngn1 (1/100; Santa Cruz Biotechnologies), antiMath1 (1/250; Chemicon), anti-Robo3 (1/100; R\&D), and anticalpain 1 (1/100; Santa Cruz Biotechnologies). Nuclei were stained with bisbenzimide (Promega) and actin with TRITC-phalloidin. Twenty-microgram cryosections were prepared from embryos fixed in $4 \%$ paraformaldehyde, embedded in gelatin $7.5 \%$ /sucrose $15 \%$, and incubated overnight at $4{ }^{\circ} \mathrm{C}$ with antibodies anti-Nrp2 (1/100; R\&D), anti-Plexin-A1 (1/100; AbCAM), and anti-Neurofilament (160 kDa, 1/100;RMO Zymed), and secondary antibodies Alexa 594, Alexa 488 (1/500; Invitrogen), and Fluoroprobe 546 (1/100) with bisbenzimide (1/1000; Promega). Chromogenic immunostaining and in situ hybridization were performed as described in Moret et al. (2007). For the t-Boc assay, $180 \mu \mathrm{M}$ fresh crosssections from embryonic spinal cords were incubated for $10 \mathrm{~min}$ with t-BOC (20 $\mu \mathrm{M}$; Invitrogen). Staining was observed immediately using a confocal microscope.

\section{Calpain inhibition in vivo}

The calpain inhibitor III (in DMSO, diluted at $12.5 \mathrm{mg} / \mathrm{kg}$; MDL28170, Calbiochem) was dissolved in saline solution $(0.9 \% \mathrm{NaCl})$ and injected intraperitoneally into pregnant mice (Charles River) at 10.5 and $11.5 \mathrm{~d}$ post-coitum (dpc). The mice were sacrificed at $12.5 \mathrm{dpc}$, and the spinal cords from embryos were collected for open book preparations and DiI labeling. Controls were performed with DMS0-treated animals.

\section{DiI staining on spinal cord open books}

Spinal cords were prepared in an open book conformation and fixed in $4 \%$ PFA for $2 \mathrm{~h}$. Small crystals of DiI (Invitrogen) were inserted in the dorsal part of one hemicord. Axon trajectories were observed using fluorescence microscopy after $48 \mathrm{~h}$.

\section{In ovo electroporation}

In ovo electroporation of chick embryos (Gallus gallus, EARL Morizeau) was performed as described previously (Moret et al. 2007). Plasmids encoding Plexin-A1, egfp, or rfp were diluted at $2 \mu \mathrm{g} / \mu \mathrm{L}$ in PBS, and at $0.5 \mu \mathrm{g} / \mu \mathrm{L}$ for Plexin-A1-PhLuorin.

\section{Analysis of calpain activity and in vitro cleavage assay}

Isolated dorsal spinal cord fresh tissue was incubated for $30 \mathrm{~min}$ at $37^{\circ} \mathrm{C}$ with control and $\mathrm{FP}^{\mathrm{cm}}$ supernatants, and was treated according to the manufacturer's instructions (Calbiochem). Calpain activity was measured by fluorogenic activity (Victor 3 multilabel counter, Perkin Elmer). Vectors encoding Plexin-A1 and FAK were transcribed and translated in vitro (TNT T7 kit, Promegal in the presence of $\mathrm{Met}^{\mathrm{S} 35}$. The products were incubated with active calpain1 (Calbiochem), and were analyzed by 
electrophoresis and autoradiography. EDTA was added in some experiments.

\section{Statistical analysis}

Statistics were done with ANOVA for quantification of PlexinA1 levels and Student's $t$-test in all other cases. $\left(^{\star \star \star}\right) P<0.0001$; $\left(^{\star \star}\right) P<0.001 ;\left(^{\star}\right) P<0.01$; (ns) not significant.

\section{Acknowledgments}

We thank J. Sanes and F. Rathien for their gift of the Sema3B and NrCAM mouse lines, respectively; Camille Charoy and Cecile Herbreteau for their help; Geraldine Gouzer for her gift of plasmid; and the Center of Quantimetry of the University of Lyon1, Rockefeller. This work is supported by CNRS, Association Française contre les Myopathies (AFM), and ANR to V.C.; and AFM fellow to H.N. K.T. is supported by NIH grant RO1NS065048. Y.Y. is supported by NIH grant RO1NS065048.

\section{References}

Abe M, Oda N, Sato Y. 1998. Cell-associated activation of latent transforming growth factor- $\beta$ by calpain. J Cell Physiol 174: 186-193.

Augsburger A, Schuchardt A, Hoskins S, Dodd J, Butler S. 1999. BMPs as mediators of roof plate repulsion of commissural neurons. Neuron 24: 127-141.

Bechara A, Nawabi H, Moret F, Yaron A, Weaver E, Bozon M, Abouzid K, Guan JL, Tessier-Lavigne M, Lemmon V, et al. 2008. FAK-MAPK-dependent adhesion disassembly downstream of L1 contributes to semaphorin3A-induced collapse. EMBO I 27: 1549-1562.

Brittis PA, Lu Q, Flanagan JG. 2002. Axonal protein synthesis provides a mechanism for localized regulation at an intermediate target. Cell 110: 223-235.

Brose K, Bland KS, Wang KH, Arnott D, Henzel W, Goodman CS, Tessier-Lavigne M, Kidd T. 1999. Slit proteins bind Robo receptors and have an evolutionarily conserved role in repulsive axon guidance. Cell 96: 795-806.

Carragher NO, Frame MC. 2002. Calpain: A role in cell transformation and migration. Int I Biochem Cell Biol 34: 15391543.

Castellani V, Chedotal A, Schachner M, Faivre-Sarrailh C, Rougon G. 2000. Analysis of the L1-deficient mouse phenotype reveals cross-talk between Sema3A and L1 signaling pathways in axonal guidance. Neuron 27: 237-249.

Chen Z, Gore BB, Long H, Ma L, Tessier-Lavigne M. 2008. Alternative splicing of the Robo3 axon guidance receptor governs the midline switch from attraction to repulsion. Neuron 58: 325-332.

Cheng HI, Bagri A, Yaron A, Stein E, Pleasure SI, Tessier-Lavigne M. 2001. Plexin-A3 mediates semaphorin signaling and regulates the development of hippocampal axonal projections. Neuron 32: 249-263.

Colamarino SA, Tessier-Lavigne M. 1995. The axonal chemoattractant netrin-1 is also a chemorepellent for trochlear motor axons. Cell 81: 621-629.

Dickson BJ, Gilestro GF. 2006. Regulation of commissural axon pathfinding by slit and its Robo receptors. Annu Rev Cell Dev Biol 22: 651-675.

Falk J, Bechara A, Fiore R, Nawabi H, Zhou H, Hoyo-Becerra C, Bozon M, Rougon G, Grumet M, Puschel AW, et al. 2005. Dual functional activity of semaphorin $3 \mathrm{~B}$ is required for positioning the anterior commissure. Neuron 48: 63-75.
Frangie C, Zhang W, Perez J, Dubois YC, Haymann JP, Baud L. 2006. Extracellular calpains increase tubular epithelial cell mobility. Implications for kidney repair after ischemia. I Biol Chem 281: 26624-26632.

Garbe DS, Bashaw GJ. 2004. Axon guidance at the midline: From mutants to mechanisms. Crit Rev Biochem Mol Biol 39: 319-341.

Gore BB, Wong KG, Tessier-Lavigne M. 2008. Stem cell factor functions as an outgrowth-promoting factor to enable axon exit from the midline intermediate target. Neuron 57: 501-510.

Hood JL, Brooks WH, Roszman TL. 2004. Differential compartmentalization of the calpain/calpastatin network with the endoplasmic reticulum and Golgi apparatus. I Biol Chem 279: 43126-43135.

Hood JL, Brooks WH, Roszman TL. 2006. Subcellular mobility of the calpain/calpastatin network: An organelle transient. Bioessays 28: 850-859.

Imondi R, Kaprielian Z. 2001. Commissural axon pathfinding on the contralateral side of the floor plate: A role for B-class ephrins in specifying the dorsoventral position of longitudinally projecting commissural axons. Development 128: 4859-4871.

Jacob TC, Bogdanov YD, Magnus C, Saliba RS, Kittler JT, Haydon PG, Moss SJ. 2005. Gephyrin regulates the cell surface dynamics of synaptic GABAA receptors. I Neurosci 25: 10469-10478.

Kadison SR, Makinen T, Klein R, Henkemeyer M, Kaprielian Z. 2006. EphB receptors and ephrin-B3 regulate axon guidance at the ventral midline of the embryonic mouse spinal cord. I Neurosci 26: 8909-8914.

Keleman K, Rajagopalan S, Cleppien D, Teis D, Paiha K, Huber LA, Technau GM, Dickson BJ. 2002. Comm sorts robo to control axon guidance at the Drosophila midline. Cell 110: 415-427.

Kidd T, Russell C, Goodman CS, Tear G. 1998. Dosage-sensitive and complementary functions of roundabout and commissureless control axon crossing of the CNS midline. Neuron 20: $25-33$.

Kruger RP, Aurandt J, Guan KL. 2005. Semaphorins command cells to move. Nat Rev Mol Cell Biol 6: 789-800.

Lustig M, Erskine L, Mason CA, Grumet M, Sakurai T. 2001. Nr-CAM expression in the developing mouse nervous system: Ventral midline structures, specific fiber tracts, and neuropilar regions. J Comp Neurol 21: 13-28.

Markgraf CG, Velayo NL, Johnson MP, McCarty DR, Medhi S, Koehl JR, Chmielewski PA, Linnik MD. 1998. Six-hour window of opportunity for calpain inhibition in focal cerebral ischemia in rats. Stroke 29: 152-158.

Mingorance-Le Meur A, O'Connor TP. 2009. Neurite consolidation is an active process requiring constant repression of protrusive activity. $E M B O$ I 28: 248-260.

Moret F, Renaudot C, Bozon M, Castellani V. 2007. Semaphorin and neuropilin co-expression in motoneurons sets axon sensitivity to environmental semaphorin sources during motor axon pathfinding. Development 134: 4491-4501.

Nishihara H, Nakagawa Y, Ishikawa H, Ohba M, Shimizu K, Nakamura T. 2001. Matrix vesicles and media vesicles as nonclassical pathways for the secretion of $\mathrm{m}$-Calpain from MC3T3-E1 cells. Biochem Biophys Res Commun 285: 845853.

Okada A, Charron F, Morin S, Shin DS, Wong K, Fabre PJ, Tessier-Lavigne M, McConnell SK. 2006. Boc is a receptor for sonic hedgehog in the guidance of commissural axons. Nature 444: 369-373.

Peltier J, Bellocq A, Perez J, Doublier S, Dubois YC, Haymann JP, Camussi G, Baud L. 2006. Calpain activation and secretion 
Nawabi et al.

promote glomerular injury in experimental glomerulonephritis: Evidence from calpastatin-transgenic mice. I Am Soc Nephrol 17: 3415-3423.

Robles E, Huttenlocher A, Gomez TM. 2003. Filopodial calcium transients regulate growth cone motility and guidance through local activation of calpain. Neuron 38: 597-609.

Sabatier C, Plump AS, Le M, Brose K, Tamada A, Murakami F, Lee EY, Tessier-Lavigne M. 2004. The divergent Robo family protein rig-1/Robo3 is a negative regulator of slit responsiveness required for midline crossing by commissural axons. Cell 117: 157-169.

Sakurai T, Lustig M, Babiarz J, Furley AJ, Tait S, Brophy PJ, Brown SA, Brown LY, Mason CA, Grumet M. 2001. Overlapping functions of the cell adhesion molecules Nr-CAM and L1 in cerebellar granule cell development. J Cell Biol 17: 1259-1273.

Shewan D, Dwivedy A, Anderson R, Holt CE. 2002. Age-related changes underlie switch in netrin-1 responsiveness as growth cones advance along visual pathway. Nat Neurosci 5: $955-962$.

Stein E, Tessier-Lavigne M. 2001. Hierarchical organization of guidance receptors: Silencing of netrin attraction by slit through a Robo/DCC receptor complex. Science 291: 19281938.

Takegahara N, Takamatsu H, Toyofuku T, Tsujimura T, Okuno T, Yukawa K, Mizui M, Yamamoto M, Prasad DV, Suzuki K, et al. 2006. Plexin-A1 and its interaction with DAP12 in immune responses and bone homeostasis. Nat Cell Biol 8: 615-622.

To KC, Church J, O'Connor TP. 2007. Combined activation of calpain and calcineurin during ligand-induced growth cone collapse. Mol Cell Neurosci 36: 425-434.

Williams SE, Grumet M, Colman DR, Henkemeyer M, Mason CA, Sakurai T. 2006. A role for Nr-CAM in the patterning of binocular visual pathways. Neuron 18: 535-547.

Wu HY, Lynch DR. 2006. Calpain and synaptic function. Mol Neurobiol 33: 215-236.

Yoshida Y, Han B, Mendelsohn M, Jessell TM. 2006. PlexinA1 signaling directs the segregation of proprioceptive sensory axons in the developing spinal cord. Neuron 52: 775-788.

Yu TW, Bargmann CI. 2001. Dynamic regulation of axon guidance. Nat Neurosci 4: 1169-1176.

Zou Y, Stoeckli E, Chen H, Tessier-Lavigne M. 2000. Squeezing axons out of the gray matter: A role for slit and semaphorin proteins from midline and ventral spinal cord. Cell 102: 363-375. 


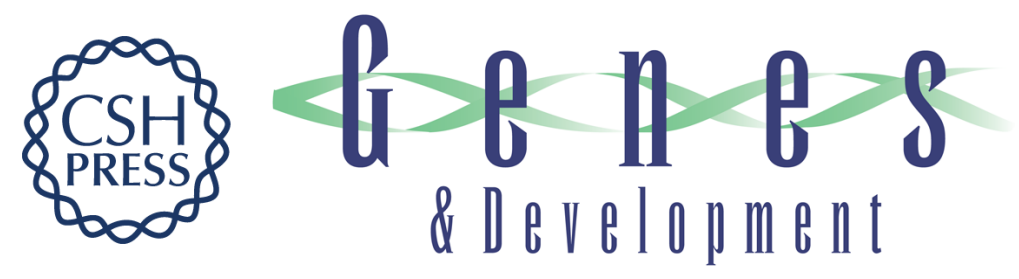

\section{A midline switch of receptor processing regulates commissural axon guidance in vertebrates}

Homaira Nawabi, Anne Briançon-Marjollet, Christopher Clark, et al.

Genes Dev. 2010, 24:

Access the most recent version at doi:10.1101/gad.542510

\section{Supplemental http://genesdev.cshlp.org/content/suppl/2010/01/26/24.4.396.DC1 Material}

References This article cites 42 articles, 9 of which can be accessed free at: http://genesdev.cshlp.org/content/24/4/396.full.html\#ref-list-1

License Freely available online through the Genes \& Development Open Access option.
Email Alerting Receive free email alerts when new articles cite this article - sign up in the box at the top Service right corner of the article or click here.

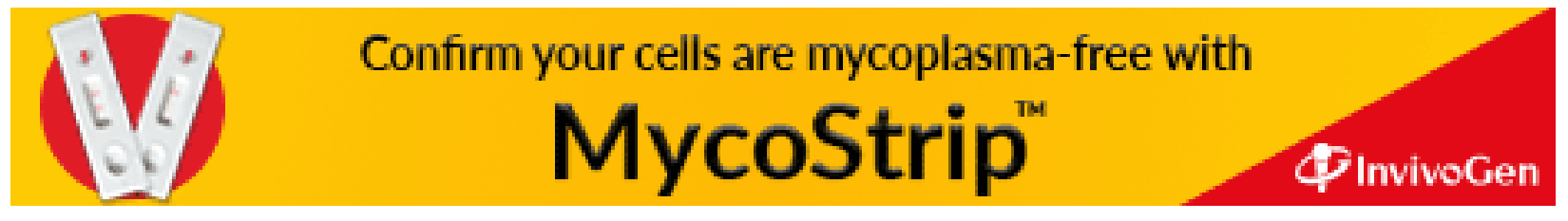

